\title{
Measuring Biomechanical Characteristics of Blood Vessels for Early Diagnostics of Vascular Retinal Pathologies
}

\author{
Nataly Yu. Ilyasova ${ }^{1}$, Alexander V. Kupriyanov ${ }^{1}$, Michael A. Ananin ${ }^{1}$, and \\ Nataly A. Gavrilova ${ }^{2}$ \\ ${ }^{1}$ Image Processing Systems Institute of Russian Academy of Sciences 151, \\ Molodogvardeiskaya st. 443001, Samara, Russia, ilyasova@smr. ru \\ ${ }^{2}$ Moscow State Medical \& Stomatological University
}

\begin{abstract}
A problem of early diagnostics of vascular pathologies is discussed. The method of diagnostic features estimation based on the mathematical model of a retina vessel fragment is presented. The experimental studies of the computa-tion accuracy of the global diagnostic parameters are considered. A technology for localization of the OD region and methods of estimating optic disk parame-ters are proposed. The methods proposed allow a differentiated diagnostics of the retinal diseases.
\end{abstract}

\section{Introduction}

Although recent decades have seen obvious advances in diagnostics and treatment of ophthalmologic diseases more people are suffering from retinal impairments of vascular genesis. Since the efficiency of treatment of vascular retinal pathologies essentially decreases with the disease progress the modern research has been focused on ways to enhance informativeness and develop maximally objective methods of early diagnostics, providing prophylactic treatment at the earliest disease stages. Nowadays, diabetes is a major medical \& social problem and among the priorities of national health services all over the world. According to the WHO, 150 million people suffer from diabetes throughout the world, with the number increasing by $6-10 \%$ every year. Among most frequent and prognostically unfavourable diseases is diabetic retinopathy (DR). Of those suffering from diabetes for over 15 years 80-97 per cent develop DR. Because the early DR stages are marked by retinal vascular changes (changes in absolute and relative diameter ratios of arteries and veins, growth of new vessels, increased vessel tortuosity, etc.) the development of digital and computer technologies for studying the retinal vascular system may show promise in early DR diagnostics. At present, the development of such technologies is associated with improvement of systems for high-quality retinal image acquisition and development of methods for quantitatively estimating the blood flow status $[1,2]$. To get a possibly full insight into the character of vascular changes at early DR stages it is expedient to combine the digital analysis of the retinal vessel routes with examination of the functional condition of the vascular wall endothelium. In this paper we introduce and discuss biomechanical characteristics of blood vessels for digital retinal image analysis, which allow the accuracy and informativeness of vascular pathology 
diagnostics to be enhanced. This paper is a further development of the methods and algorithms for estimating the geometric parameters of vessels proposed in Ref. [3]. Reference [4] deals with estimating one of the parameters (beading) but uses a different approach to extracting vessels from the image.

\section{Formation of Retinal Blood Vessels Diagnostic Parameters}

To measure the biomechanical vessel characteristics we took a totally new approach to analyzing the retinal image via a tracing vessel segmentation. The method employs a scanning polar frame and allows us to calculate local vessel features (diameter and direction at each point). To determine a set of vascular characteristics and enhance the measurement accuracy we have developed a mathematical model of the vessel (Fig.1) that is defined by the following functions: $x=x(t), y=y(t), r=r(t), 0 \leq t \leq L_{v}$, where $x(t), y(t) x=x(t), y=y(t), r=r(t), 0 \leq t \leq L_{v}$, where $x(t), y(t)$ are differentiable functions defining a center line hereafter called the route; $r(t)$ is the branch thickness function (the distance from the route to the vessel boundary reckoned along the perpendicular to the route); $t$ is the distance from the route initial point measured along the route; and $L_{v}$ is the route length.

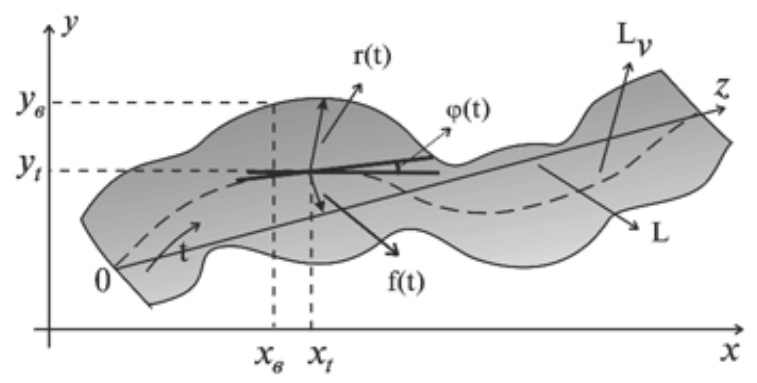

Fig. 1. Mathematical model of a retina vessel fragment

These characteristics uniquely define the route direction function $\varphi(t)$ at each point; the local height function $f(t)$ defined by the distance from the route current point to its projection onto a segment L connecting the initial and final route points (Fig.2); and the configuration of the vessel boundaries hereafter called the walls $x_{1}^{b}=x_{1}^{b}(t)$, $y_{1}^{b}=y_{1}^{b}(t), x_{2}^{b}=x_{2}^{b}(t), y_{2}^{b}=y_{2}^{b}(t), 0 \leq t \leq L_{v}$.

The local features involve the route, as well as the distribution of the vessel diameter and the direction along the route. These are calculated immediately from the image using an algorithm for vessel tracing (central line tracing) [3]. The global features include the average diameter, linearity, beading, thickness variation amplitude, thickness variation frequency, thickness tortuosity, route variation frequency, route variation amplitude, and route tortuosity. These serve to characterize the entire vessel on the whole and are later used as diagnostic features. 
The vessel average diameter $\bar{D}$ is derived from

$$
\bar{D}=2 \bar{r}=\frac{2}{N} \sum_{n=1}^{N} r\left(t_{n}\right),
$$

where $t_{n}=n \Delta, N$ is the number of pixels of a local radius measured along the vessel at reliable points, and $\bar{r}$ is the vessel average radius.

The vessel linearity $\mathrm{P}$ characterizes the vessel deviation from a straight line and is defined as the ratio of length $\mathrm{L}_{\mathrm{v}}$ of the vessel medial line to the length $\mathrm{L}$ of a straight line connecting the initial and final points of the route:

$$
P=L_{v} / L=\sum_{n-1}^{N-1} \sqrt{\left(x_{n}-x_{n+1}\right)^{2}+\left(y_{n}-y_{n+1}\right)^{2}} / \sqrt{\left(x_{1}-x_{N}\right)^{2}+\left(y_{1}-y_{N}\right)^{2}} .
$$

The vessel beading $\mathrm{S}$ characterizes the branch thickness irregularity and is defined as the ratio of the root-mean-square deviation of the vessel radius to its average value:

$$
S=\sqrt{\overline{r^{2}}-\bar{r}^{2}} / \bar{r}
$$

where $\bar{r}$ is the vessel average radius and $\overline{r^{2}}$ is the radius mean square.

The vessel thickness variation amplitude $A_{0}$ characterizes the deviation of the vessel walls from a straight line and is defined as

$$
A_{0}=\sqrt{2 \overline{r^{2}}-2 \bar{r}^{2}} \text {. }
$$

The vessel thickness variation frequency $\omega_{0}$ characterizes a change in the wall direction per unit length and is defined as

$$
\omega_{0}=\frac{2 \pi}{N} m_{0}, \quad m_{0}=\arg \left(\max _{1<m<N} R(m)\right), \quad R(m)=\left|\sum_{n=0}^{N-1} r\left(t_{n}\right) \exp \left(-i \frac{2 \pi n m}{N}\right)\right|,
$$

$m_{0}$ is the number of maximal value of Fourier-spectrum of the thickness function.

The thickness tortuosity $I_{0}$ characterizes the rate of change of the thickness function along the route approximated by a harmonic function of amplitude $A_{0}$ and frequency $\omega_{0}$ and is derived from $I_{0}=A_{0} \omega_{0}$.

The route tortuosity $I_{1}$ characterizes the rate of change of the route function at a selected segment, which is approximated by a harmonic function of amplitude $A_{1}$ and frequency $\omega_{1}$, defined as $I_{1}=A_{1} \omega_{1}$, and derived from

$$
P=\frac{2}{\pi} \sqrt{1+I_{1}^{2}} \cdot E(k), \quad k=I_{1} / \sqrt{1+I_{1}^{2}},
$$

where $\mathrm{P}$ is the branch linearity, and $E(k)$ is the total elliptic integral of the $2^{\text {nd }}$ kind. 
The route variation amplitude $A_{1}$ characterizes the degree of deviation of the route trajectory from the straight line and is defined as

$$
A_{1}=2 \bar{f} \cdot E(k) /\left(1+\ln \left(I_{1}+\sqrt{1+I_{1}^{2}}\right) /\left(I_{1} \sqrt{1+I_{1}^{2}}\right)\right),
$$

where $I_{1}$ is the route tortuosity and $\bar{f}$ is the vessel average height.

The vessel fragment route variation frequency $\omega_{1}$ characterizes how often the direction of the branch is changed per unit length and is defined as

$$
\omega_{1}=I_{1} / A_{1} \text {. }
$$

Given below are the experimental studies of the computation accuracy of the following four global parameters: route variation amplitude, route variation frequency, thickness variation amplitude, and thickness variation frequency. In studying the route parameters, images of ideal routes with their trajectories defined as sinusoidal functions of different frequency and amplitude were generated (Table 1). The error in determining the route frequency is caused by the error introduced by the branch tracing algorithm. In studies of the thickness parameters, images of ideal routes with trajectories of their boundaries defined as sinusoidal function of different frequency and amplitude were generated (Table 2). The error in constructing the parameter estimates is caused by the effect of route image discretization.

The studies conducted have shown that the above-discussed features can be used for assessment of the general retinal pathology.

Table 1. The results of the experimental studies of the methods for estimating the route parameters in test images

\begin{tabular}{lllll}
\hline Route & Amplitude & \multicolumn{2}{l}{ Frequency } \\
\hline & Ideal & Estimated & Ideal & Estimated \\
10 & 10 & 10.13 & 1.5 & 1.500 \\
& 10 & 10.26 & 2.0 & 1.789 \\
20 & 21.10 & 2.5 & 2.049 \\
& 20 & 20.16 & 4.5 & 4.127 \\
\end{tabular}

Table 2. The results of the experimental studies of the methods for estimating the route thickness parameters in test images

\begin{tabular}{lllll}
\hline Route & Amplitude & & Frequency \\
\hline & Ideal & Estimated & Ideal & Estimated \\
& 2.5 & 2.430 & 3.0 & 3.002 \\
& 8.0 & 7.918 & 2.5 & 2.441 \\
& 2.0 & 2.102 & 12.5 & 12.478 \\
& 7.0 & 7.041 & 9.5 & 9.482 \\
\hline
\end{tabular}




\section{Methods for Estimating Optic Disk Parameters}

In the literature there are numerous reports about optic disk impairments caused by the elevated intraocular pressure. The system to autoregulate circulation in the blood vessels feeding the optic disk (OD) is a key mechanism that supports the normal circulation in the optic nerve $(\mathrm{ON})$ head. Disturbance of this function is a risk factor in the progressive development of glaucoma-related ON atrophy $[5,6]$.

Decreased OD vascularization in glaucoma patients and atrophic changes in peripapillar vessels confirm that vascular pathologies have their part in development of optical neuropathy. In this connection, ophthalmologists doing their research with Moscow State Medical \& Stomatological University (Ophthalmology Department) commissioned our team to estimate the OD diagnostic parameters. The key characteristics of vascular pathology are the ratio of the total diameter of thin vessels on the OD edge to its perimeter, as well as the total area of the aforementioned vessels in the OD region.

\subsection{Localization of the OD}

The automated system developed for OD analysis involves the following stages of retinal image processing: localization of the OD region, profiling along the OD edge, vessel detection on the OD edge, estimation of the vessel local features (direction and diameter), and calculation of the diagnostic parameters. The pre-processing stage is aimed at obtaining a binary image of the OD region and determining parameters approximating the OD edge contour.

Based on the data of the original full-color retinal image and the OD contour parameters we can build a profile along the OD edge and perform the subsequent processing using a smoothing filter. The algorithm for obtaining a binary image can be broken down into three stages: profiling the brightness function to get a binary preparation, rank filtration of the binary image, and elimination of large noise fragments (Fig.2).

To obtain the OD edge parameters we used an algorithm that approximates the OD edge by an ellipse of minimal area that covers the whole (or nearly whole) area OD region. The equation of an ellipse with an arbitrary center and tilt is given by

$$
\frac{\left(\left(x-x_{0}\right) \cos \varphi-\left(y-y_{0}\right) \sin \varphi\right)^{2}}{a^{2}}+\frac{\left(\left(y-y_{0}\right) \cos \varphi+\left(x-x_{0}\right) \sin \varphi\right)^{2}}{b^{2}}=1 .
$$

A five-dimensional parallelepiped defining the range of values of the parameters $a$, $b, x_{0}, y_{0}$ and $\varphi$ is broken down into cells. In the grid nodes, the area is calculated and the inclusion condition is checked.

The major stages of the algorithm are: (1) exhaustive search of the values $x_{0}, y_{0}, \varphi$; (2) for a given triplet $\left(x_{0}, y_{0}, \varphi\right)$, determination of the semi-axes $(a, b)$ of a minimalarea ellipse covering the OD region. The result of the OD region localization and obtaining the ellipse parameters is shown in Fig.2. 

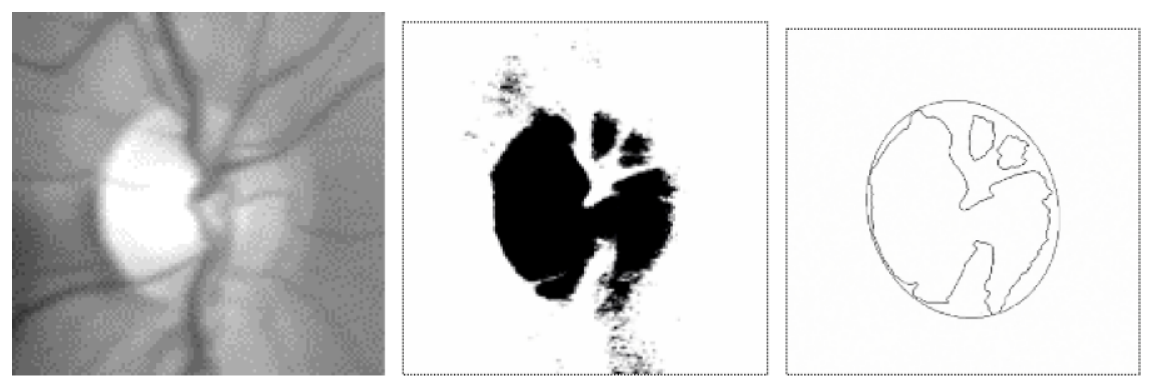

Fig. 2. Operation of the algorithm for the OD region localization: original image; view after brightness profiling; and the OD region contour and the approximating ellipse

\subsection{Vessel Detection and Parameter Estimation on the OD Edge}

The retinal vessel detection was performed on the basis of analysis of the gray-level profile represented as a brightness function scan along the derived contour of the OD region. In pre-processing, one of the problems consists in bringing the color image profile to the gray-level through transforming the R-, G-, and B-pixels of the original image into the corresponding brightness values. In the course of experimental studies, the red and green components were found to carry a major bulk of information about the background and vessels. Because of this, the arithmetic mean of the red and green components was used as a resulting value of brightness of the current profile point. To suppress noise an averaging filter was used.

The method for vessel detection on the OD edge is based on the analysis of local minima of the derived profile brightness function. The procedure involved a sliding local approximation by a second-order polynomial. The search for minimums corresponding to the vessel centers is carried out analytically, with the subsequent analysis aimed at discarding false minimums. In the algorithm of thickness estimation, the vessel boundaries are given by the profile inflection points nearest to the center. An average relative shift of vessel centers on $\mathrm{N}$ neighboring ellipses (similar to the original one, with the coefficient close to 1) allows us to find the vessel direction. The averaging is conducted in the vessel center neighborhood on the major profile. The estimate of vessel thickness is corrected by multiplying by the cosine of the angle between the derived vessel direction and the normal to the OD contour, because the vessels are not perpendicular to the OD region edge.

\subsection{Experimental Studies of the Methods}

The performance of the algorithm for estimating the vessel local features was checked using test and original OD images. In the course of studies, we examined how the algorithm parameters affected the accuracy of vessel detection and accuracy of diameter estimation on the OD edge. Out of all the experiments conducted, below we 
discuss only those looking into the impact of noise on the diameter estimation accuracy in test images (Fig.3). The algorithm in question (scheme 1) was compared with the algorithm in which the vessel direction is being sought by using a fan transform [7] (scheme 2). It can be seen from Fig.3 that noise begins to affect the diameter estimation accuracy at the noise-to-signal ratio of 0.216 , suggesting that the method shows good robustness.

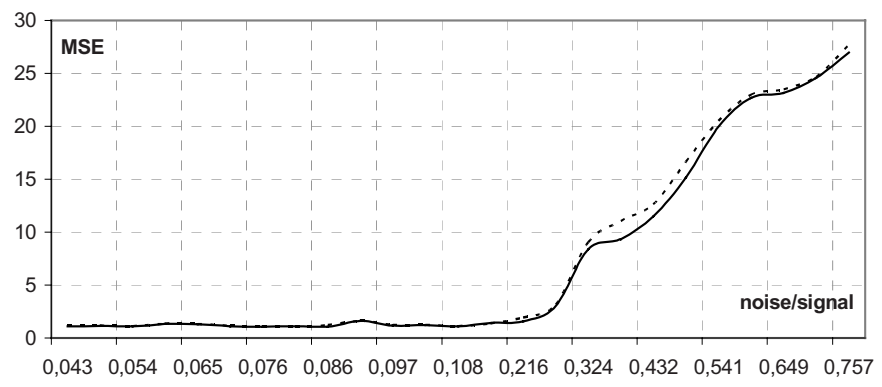

Fig. 3. The error of the vessel thickness estimation vs noise level (solid line - scheme 1, dotted - scheme 2)

Figure 4 shows the result of the algorithm operation for localization of the OD region, contouring and estimating the OD local parameters in an original image. The interface of the OD analysis system allows simultaneously viewing all the necessary data and the results of studies.

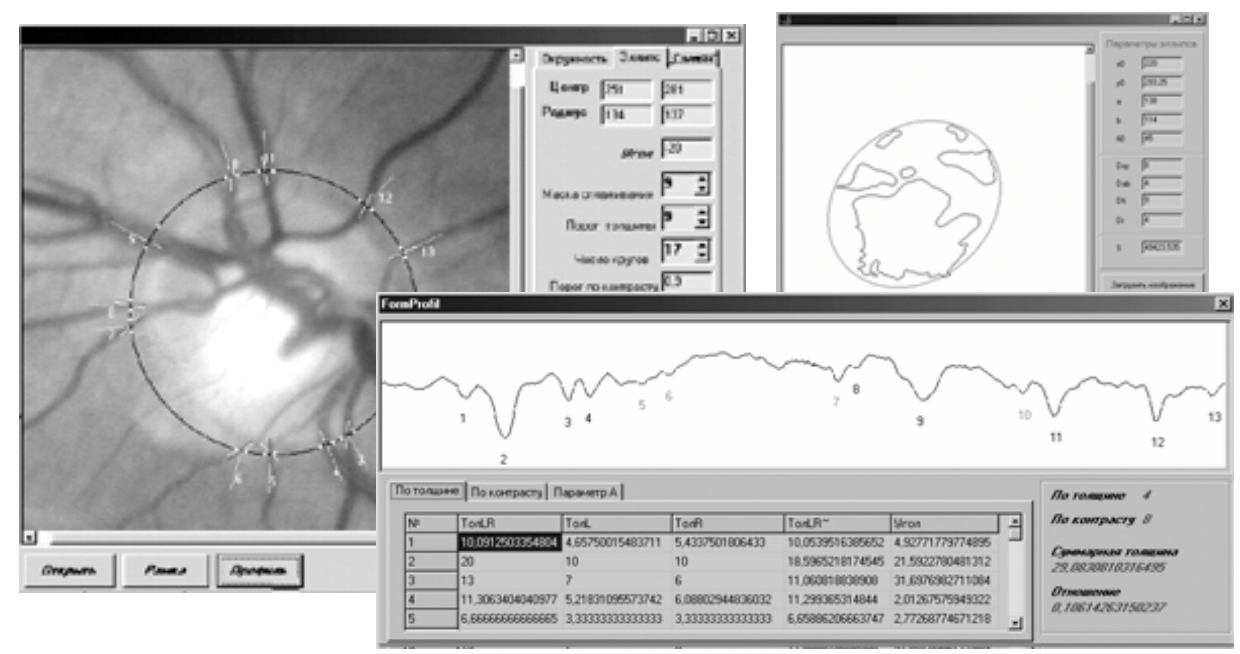

Fig. 4. Interface of the OD analysis system (the result of operation of the algorithms for the OD region localization, contouring, and estimating the vessel local features in the original image) 


\section{Conclusions}

We have developed biomechanical blood vessel characteristics for digital analysis of the retina, which aim to enhance the accuracy and informativeness of vascular pathology diagnostics. The biomechanical characteristics essentially facilitate the expert estimate of the vascular pathology, being capable of detecting slightest pathological changes, its degree, and development probability. The estimation methods discussed have formed the basis for a computerized system for measuring the geometric parameters of biomedical images [3]. The system allows the objective quantitative results to be derived and extends the capabilities of the existing medical methods. The analysis system also includes methods and algorithms for estimating the OD geometric characteristics and a method of searching for vessels on the OD edge and localizing the OD region using peculiarities of the OD color-brightness characteristics and the OD region approximation contouring. Introduction of the developed methods into medical use will enhance its capabilities and allow automatic diagnostics of some diseases and monitoring of pathological retinal changes on the basis of objective quantitative data.

Acknowledgements. This work was financially supported by the U.S. Civilian Research \& Development Foundation (CRDF) and the Ministry of Education \& Science of the Russian Federation (CRDF project REC-SA-014-02) as part of the Basic Research \& Higher Education (BRHE) program; by the Russian Foundation for Basic Research grant under № 03-01-00642; and by the Human Capital Foundation grant.

\section{References}

1. Jomier, J., Wallace, D.K., Aylward, S.R.: Quantification of Retinopathy of Prematurity via Vessel Segmentation. Proceedings of MICCAI 2003, LNCS 2879 620-626.

2. Osareh, A., Mirmehdi M., Thomas B., Markham R.: Classification and Localisation of Diabetic-Related Eye Disease. ECCV 2002, LNCS 2353 502-516

3. Ilyasova, N.Yu., Ustinov A.V., Baranov V.G.: An Expert Computer System for Diagnosing Eye Diseases from Retina Images. Optical Memory and Neural Networks, Vol. 9, No. 2 (2000) 133-145

4. Ching-Wen Yang, Dye-Jyun Ma, Shuenn-Ching Chao, Chuin-Mu Wang, Chia-Hsin Wen, Chien-Shun Lo, Pau-Choo Chung, Chein-I Chang: Computer-aided diagnostic detection system of venous beading in retinal images, Optical Engineering, Vol.39, No.5, 2000, pp.1293-1303

5. Mendels, F., Heneghan, C., Thiran, J.P.: Identification of the Optic Disk Boundary in Retinal Images Using Active Contours. Proceedings of the Irish Machine Vision and Image Processing Conference (1999) 103-115

6. Chanwimaluang, T., Fan, G.: An Efficient Algorithm for Extraction of Anatomical Structures in Retinal Images, Proc. IEEE International Conference on Image Processing, Barcelona, Span, September 2003

7. Baranov, V.G., Khramov, A.G.: Discrete fan-shaped Radon transform for net-like structures' centerlines detection. Journal "Computer Optics", Vol. 23 (2002) 44-47 